\title{
The Usefulness of the Energy Harvested from Additive Noises in Power Line and Wireless Media
}

\author{
Victor Fernandes, Thiago R. Oliveira, and Moisés V. Ribeiro
}

\begin{abstract}
Aiming to come up with strategies for harvesting energy in hybrid power line communication (PLC)-wireless systems, this work focuses on the amount of energy that can be potentially harvested from the additive noise in electric power grids and in the air within residential facilities when the frequencies from 1.7 up to $100 \mathrm{MHz}$ are taken into account. Based on statistical analyses of a residential data set, we show that the average power of the measured additive noise in the power line is higher in the morning than other periods of the day. Also, it is higher than the values from the energy harvested from in the air, in the same frequency band. Moreover, we show that the amount of energy harvested from these additive noises can yield achievable data rates between hundreds of kbps up to dozens of Mbps if hybrid PLC-wireless channels are used for data communication purposes.
\end{abstract}

Index Terms-Achievable data rate, energy harvesting, power line communication, wireless communication.

\section{INTRODUCTION}

$\mathbf{E}$ NERGY harvesting $(\mathrm{EH})$ has emerged as one of the promising solutions in the context of green communications. The possibility of allowing the reuse of a wasted resource can exert a pivotal role in advancing self-sustainable telecommunication networks. As stated in [1], EH allows the savings of non-renewable energy and also makes the communication media environmentally friendly. In the context of radio-frequency $(\mathrm{RF}), \mathrm{EH}$ can be subdivided into two distinct approaches named as ambient or dedicated, according to the power source [2]. In the former approach, a device harvests its energy from an ambient source of power, which can be the RF signal irradiated by analog or digital $\mathrm{TV}$, radio station (e.g., amplitude modulation (AM) and frequency modulation (FM)), WiFi and Bluetooth devices, cellular base stations, among others. On the other hand, the latter approach aims at powering a particular device by means of directional power transfer.

Nowadays, the opportunity of harvesting energy from wireless communication (WLC) sources, based on EH strategies,

This work was in part supported by the Coordenação de Aperfeiçoamento de Pessoal de Nível Superior - Brasil (CAPES) - Finance Code 001, FINEP, FAPEMIG, CNPq, and INERGE.

V. Fernandes (e-mail: victor.fernandes@engenharia.ufjf.br) is with the Department of Electrical Engineering, Federal University of Juiz de Fora, Juiz de Fora 36036 330, Brazil, and also with the Electrical Engineering Department, Centro de Ensino Superior de Juiz de Fora (CES/JF), Campus Academia, Juiz de Fora 36016 000, Brazil.

T. R. Oliveira (thiago.oliveira@ifsudestemg.edu.br) is with the Electronics Department, Federal Institute of Education, Science and Technology of the Southeast of Minas Gerais (IFSEMG), Campus Juiz de Fora, Brazil.

M. V. Ribeiro (mribeiro@engenharia.ufjf.br) is with the Department of Electrical Engineering, Federal University of Juiz de Fora, Juiz de Fora 36036 330, Brazil.

Digital Object Identifier: 10.14209/jcis.2020.6 has been investigated due to the possibility of creating wireless self-sustainable networks [2], [3]. However, [1] showed the importance of electric power grids when the uninterrupted delivery of energy is necessary. In this context, [4] related the power line communication (PLC) noise impulsiveness with the harvested energy amount while [5] studied the energy harvesting potential when the induced signal that is radiated from the power lines is taken into account. Also, [6] demonstrated the usefulness of the medium-voltage PLC additive noise in urban and rural environments as well as [7] exploited the diversity of both wired and wireless media for EH purpose and showed that they can be used in tandem for improving achievable data rates of low-bit-rate devices.

Due to the spectrum scarcity and the necessity of maximizing the use of channel resources, the (concatenated) hybrid PLC-WLC channel was introduced in [8]. As shown in Fig. 1, this channel is composed of unshielded power cables of the power grid and the air. Thus, a PLC device sends a signal carrying information that partially radiates from the power cable and, as a consequence, it can be sensed by a WLC device operating in the same frequency band, defining the PLC $\rightarrow$ WLC direction. On the other hand, a signal transmitted by a WLC device can be irradiated into the power cable and, as a consequence, the induced signal is received by a PLC device through a PLC coupler, which defines the WLC $\rightarrow$ PLC direction. This kind of concatenated data communication medium can be seen as a way of introducing mobility in PLC systems. The use of data communication between WLC and PLC devices defines the so-called hybrid PLC-WLC systems.

None of the previous works considered a concatenated version of the hybrid PLC-WLC channel for data communication when the ambient $\mathrm{EH}$ is exploited in each medium (power cable and air), for feeding the transceivers. As well, the frequencies which are usually considered for data communication in broadband-PLC (up to $100 \mathrm{MHz}$ ) are assumed to be used for EH purposes. Furthermore, this work is based on a data set of measured additive noise samples, which is usually not considered in the majority of the works about EH.

This work focuses on the evaluation of the suitability of EH from PLC and WLC additive noises inside residences for data communication purposes through hybrid PLC-WLC systems [8], [9]. These analyses are based on a set of measured additive noises, which were acquired from the power cable (PLC additive noise) and the air (WLC additive noise) measurement campaign [10]. Furthermore, the amount of energy that can be harvested from the additive noise in these media is used to evaluate achievable data rates in hybrid PLC-WLC systems. Numerical results show that recycling the wasted energy in 


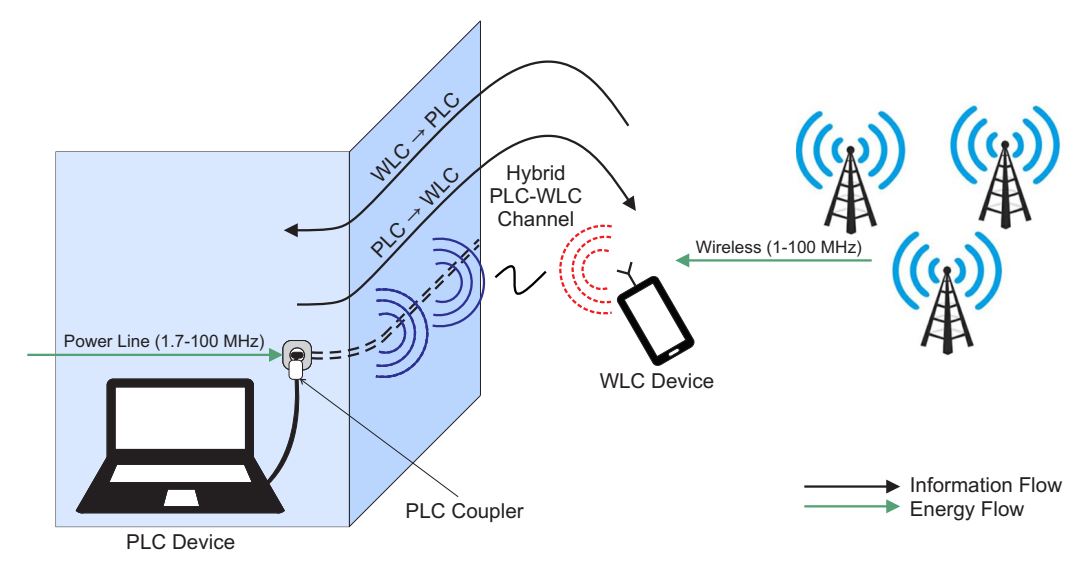

Fig. 1: A typical concatenated hybrid PLC-WLC system.

the additive noises (i.e., harvesting the energy associated with the additive noise in PLC and WLC media) can result in data rates suitable for several applications related to smart grids, IoT, and Industry 4.0.

\section{Measurement Data Sets}

The numerical analyses of the harvested energies are carried out with a data set composed of measures of additive noises, which was obtained from a measurement campaign carried out to measure the additive noise in the power lines and the air in in-home facilities. This data set was originally applied to analyze the PLC channel in the context of the spectrum sensing problem [10] in the frequencies of up to $100 \mathrm{MHz}$. The measurement campaign was carried out in 16 distinct residences spread over the urban area of Juiz de Fora, Brazil. Inside each residence, 300 measures of the additive noises in the power line and in the air were obtained. This amount of measures was divided equally into three different periods of the day: morning, afternoon and night. Each of 4, 800 measures of the additive noise in both media (PLC and wireless) is converted to an average power value (in Watts as the power dissipated by a load of $1 \Omega$ ) by using

$$
P_{M}=\frac{\eta}{T_{A N}} \int_{T_{A N}}\left|V^{q}(t)\right|^{2} d t
$$

where $V^{q}(t)$ is the additive noise signal associated with the $q^{\text {th }}$ data communication direction, in which $q \in$ $\{\mathrm{PLC} \rightarrow$ WLC, WLC $\rightarrow$ PLC $\}$ denotes the PLC to WLC and PLC to WLC directions, respectively; $T_{A N}=40.96 \mu \mathrm{s}$, which is the multiplication of 8192 samples by $T_{s} ; T_{s}=1 / f_{s}$ is the sampling period and $f_{s}=200 \mathrm{MHz}$ denotes the adopted value of the sampling frequency for carrying out the measurement campaign; and $0<\eta \leq 1$ is the RF-to-DC conversion efficiency [2].

Assuming that the additive noise is a colored and Gaussian random process, the frequency band from $1.7 \mathrm{MHz}$ up to $100 \mathrm{MHz}$, and the hybrid PLC-WLC channels are frequency selective [8], then channel frequency responses (CFRs) estimates and additive noise sequences are combined with the average power provided by (1) to calculate the achievable data rate in the hybrid PLC-WLC by using

$$
C=\max _{\varrho^{q}(f)} \int_{B_{w}} \log _{2}\left(1+\frac{\varrho^{q}(f)\left|H^{q}(f)\right|^{2}}{S_{V}^{q}(f)}\right) d f,
$$

subject to $\int_{-B_{w}}^{B_{w}} \varrho^{q}(f) d f \leq P_{M}$, where $\varrho^{q}(f)$ is the power spectrum density (PSD) of the transmitted signal's power mask; $\left|H^{q}(f)\right|^{2}$ is the CFR squared magnitude associated with the $q^{t h}$ data communication direction. Further, $B_{w} \approx$ $100 \mathrm{MHz}$ is the frequency bandwidth and $S_{V}^{q}(f)$ refers to the PSD of the additive noise at the receiver of the $q^{\text {th }}$ data communication direction. Further, we consider that the full channel state information (CSI) is available and the optimal power allocation is applied in $\varrho^{q}(f)$ by means of the waterfilling (WF) technique [11].

The data set related to the estimates of hybrid PLC-WLC channels were obtained from a measurement campaign performed in 7 residences in Juiz de Fora, Brazil [8], [12]. It is important to highlight that this data set was acquired from distinct time and places from the previous one. The CFRs of these channels are estimated by applying digital signal processing in accord with [13]. In the adopted channel estimation methodology, a sounding signal is injected into the power line, through a PLC coupler [14], and its distorted version is extracted from the air by using an antenna. In this sense, two different scenarios were taken into account according to the distance between the outlet in which the estimation signal was injected in the power line and the antenna used to measure the received signal. The former scenario, which is associated with the so-called short-path (SP) channels, covers the distances from 0 up to 2 meters and comprise those channels with the lowest attenuation profile while latter scenario, which refers to the so-called long-path (LP) channels, covers distances between 2 and 6 meters and encompasses the channels with high attenuation and distortions. During the measurement campaign of the PLC-WLC channels, 200 and 93 different combinations of outlet and antenna positions were considered for SP and LP channels, respectively. From each combination, 600 consecutive estimates of CFRs were obtained, resulting in a total of 175,428 CFRs estimates. Also, 52 and 60 measures of the additive noise in the power line and in the air, respectively, were obtained. The length of a single sequence of measured additive noise is constituted by 3,000, 000 samples. 


\section{NumericAl Results}

The complementary cumulative distribution function (CCDF) of the 4,800 values of average power of PLC and WLC additive noises are shown in Fig. 2. In this plot, we adopt $P_{M}[\mathrm{dBmW}]=10 \log _{10}\left(P_{M}[\mathrm{~W}]\right)+30$. As can be seen, the average power in PLC additive noise is higher than in WLC one. For instance, $80 \%$ of the PLC additive noises in power lines show values of average power higher than $-9 \mathrm{dBmW}$, while, for the WLC case, this value is higher than $-17 \mathrm{dBmW}$. Also, the maximum value of average power observed in the PLC additive noise is of approximately $29 \mathrm{dBmW}$, against approximately $11 \mathrm{dBmW}$ for the WLC additive noise.

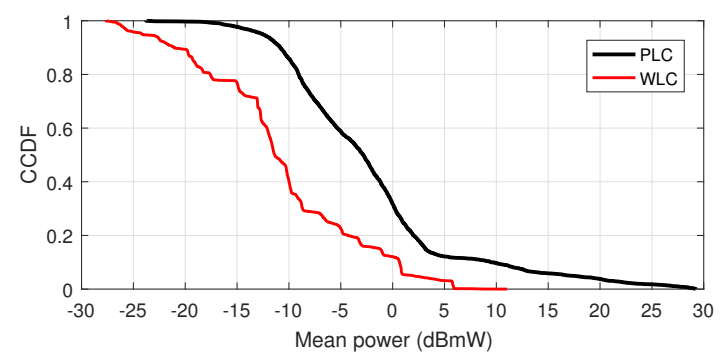

Fig. 2: The average power of PLC and WLC additive noises obtained with the measured data sets.

The CCDF of average power in the PLC and WLC additive noises, considering different periods of the day, can be seen in Fig. 3. In the PLC scenario, the average power of the additive noise is higher in the morning than in other periods of the day. This is related to the dynamic of the loads which are connected to the electric power grid. Also, this behavior suggests that the users of the measured places are using more electricity during the morning period, which coincides with the daily house cleaning routine. It is important to mention that it can vary depending upon the profiles of energy consumers. On the other hand, the average power of the additive noise in the WLC channel is more uniform in the different periods of the day due to the randomness and the large number of the various sources of WLC additive noise. For instance, AM and FM radio broadcast signals, and other telecommunication systems operating in the chosen frequency band constitute the measured WLC additive noise.

The PSDs of the additive noise data sets discussed in [10] are shown in Fig. 4. As expected, the PLC additive noise has more power in the low frequencies, while the PSD of the WLC additive noise is more uniformly distributed within the chosen frequency band, which agree with the theory. According to this plot, the difference between PLC and WLC additive noises' average power reaches a peak of $36.11 \mathrm{dBm}$, approximately, in $1.5 \mathrm{MHz}$, against to the minimum value of $0.02 \mathrm{dBm}$ in $71.4 \mathrm{MHz}$. Also, as can be noticed, the signals yielded by wireless stations are induced into power line cables ${ }^{1}-$ the induced part of these signals in the power lines are usually named as narrow-band noise. Also, it is relevant to see that, for instance, through some peaks observed in the PSD of the PLC

\footnotetext{
${ }^{1}$ The power cable used in the electric power systems are unshielded and, as a consequence, it works as an antenna
}
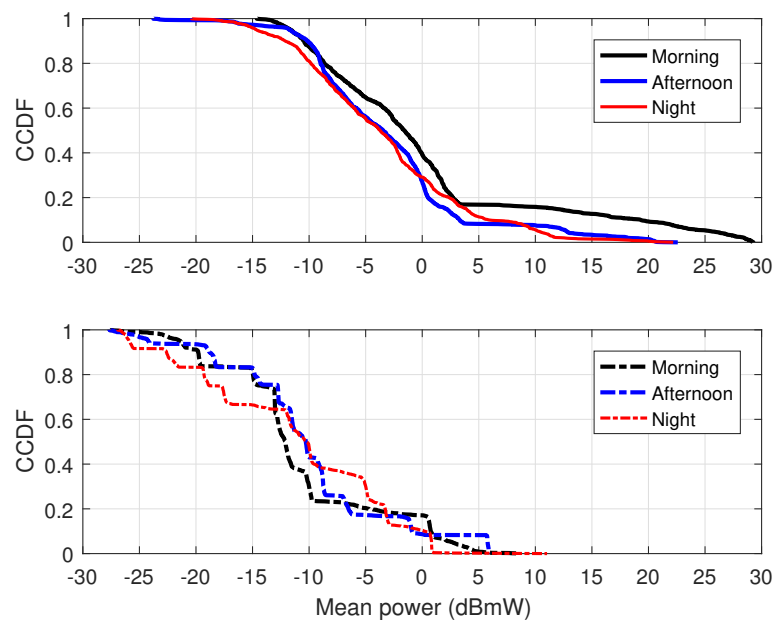

Fig. 3: The average power of the PLC (top) and WLC (bottom) additive noises per period of the day.

additive noise just below $100 \mathrm{MHz}$, in which FM broadcasting radio signals prevail over other components of the PLC and WLC additive noises.

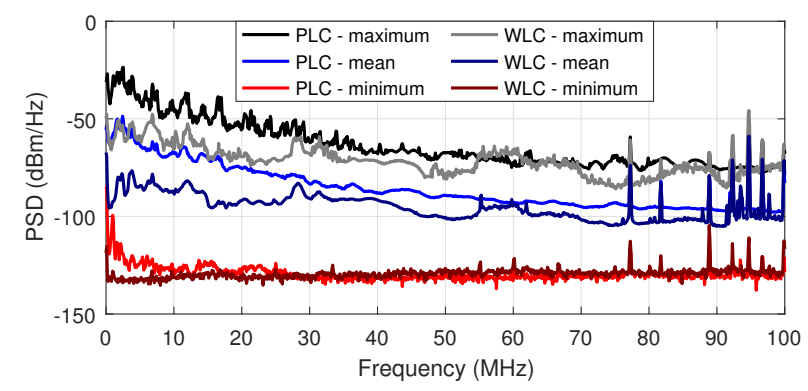

Fig. 4: PSD of the PLC and WLC additive noises.

\section{A. Achievable Data Rate}

This subsection deals with the theoretical achievable data rate that can be obtained by considering the following cases:

- Case \#1: a PLC device that harvests the energy from the PLC additive noise and communicates through the hybrid PLC-WLC channel in the PLC to WLC direction.

- Case \#2: a WLC device which harvests the energy from the WLC additive noise and communicates through the hybrid PLC-WLC channel in the WLC to PLC direction.

To this end, we assume that the source of power (i.e., the additive noise) is uninterruptible. Also, we use $\eta=\{1,0.5\}$ to investigate the situations which constitutes an ideal scenario $(\eta=1)$ and a practical scenario $(\eta=0.5)$. Furthermore, [8] showed that both data communication path directions result in similar CFR estimates. Thus, the differences between cases $\# 1$ and \#2 is the amount of harvested energy at the transmitter device and the PSD of the receiver's additive noise in each possible communication direction.

The CCDF of the achievable data rates obtained in cases \#1 (top) and \#2 (bottom) are shown in Figs. 5 and 6 for the SP and LP channels, respectively, when two distinct values of $\eta$ apply. By comparing these figures, we can notice that the SP channels offer higher values of achievable data rates than LP ones in both cases. Also, as can be seen in both figures, 
the case \#1 achieves a better performance than the case \#2 in terms of achievable data rate due to the high power nature of PLC additive noise. For instance, at Fig. 5 and assuming $\eta=$ $0.5,80 \%$ of the data sets yielded achievable data rates greater than $27 \mathrm{Mbps}$ and $4 \mathrm{Mbps}$ in cases \#1 and \#2, respectively. Looking at Fig. 6 (LP channels), using the same values of $\eta$ and other aforementioned assumptions result in achievable data rates greater than $310 \mathrm{kbps}$ and $20 \mathrm{kbps}$ in cases \#1 and $\# 2$, respectively.
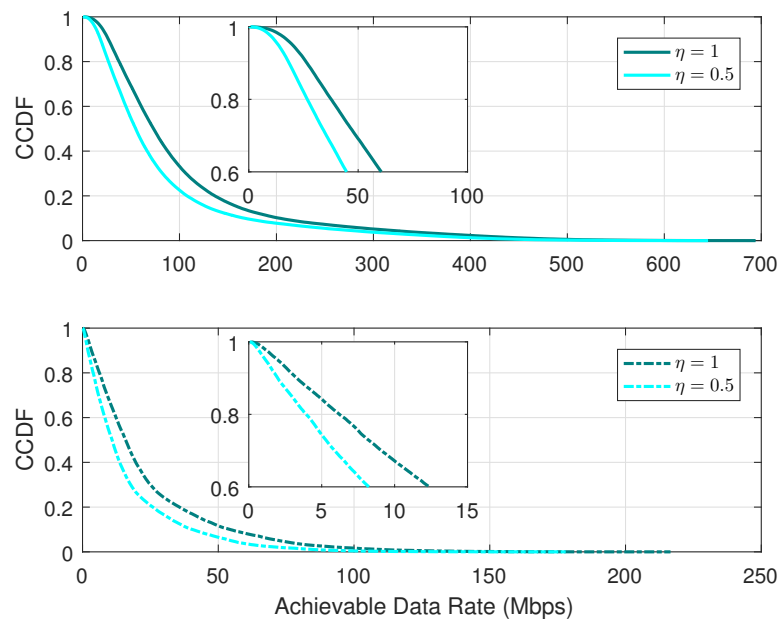

Fig. 5: Achievable data rates: The CCDF for the case \#1 (top) and the case \#2 (bottom) when SP channels apply.
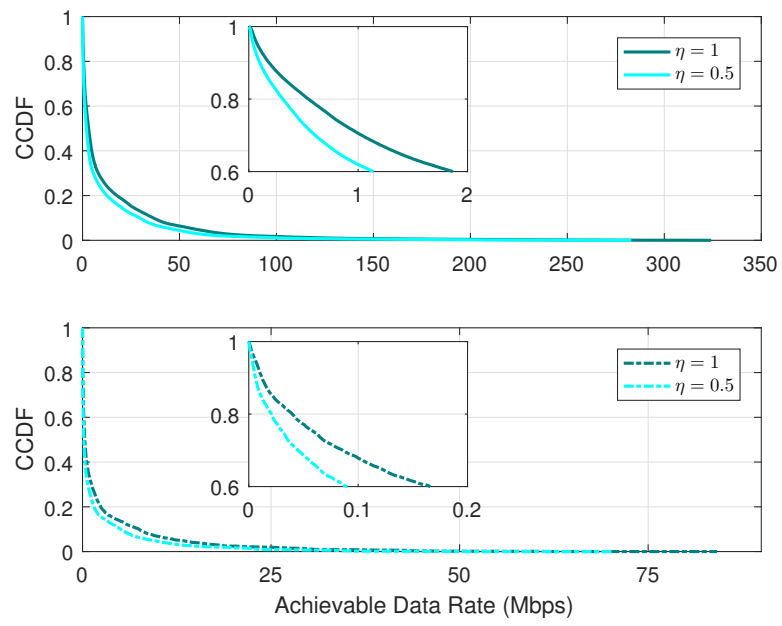

Fig. 6: Achievable data rates: The CCDF for the case \#1 (top) and the case \#2 (bottom) when LP channels apply.

Another interesting value is the maximum data rate. In accord with Fig. 5 (SP channels and $\eta=1$ ), case \#1 the maximum data rate is $694 \mathrm{Mbps}$ while case \#2 is $216 \mathrm{Mbps}$. For the same value of $\eta$ and LP channels (Fig. 6), the maximum data rates are $324 \mathrm{Mbps}$ and $84 \mathrm{Mbps}$ in cases $\# 1$ and \#2, respectively. This comparison also supports the previous paragraph's statements.

Aiming to capture the influence of the noise in distinct periods of the day, the CCDFs of the achievable data rates yielded in cases \#1 and \#2 during the morning, afternoon, and at night are shown in Fig. 7 for the SP channels (top) and for the LP channels (bottom) when $\eta=1$. In this figure, it is clear that case \#1 is better than case \#2 for both SP and LP channels at any period of the day. Here, the same trends observed in Fig. 3 (top) can be seen in both SP and LP channels for case \#1, i.e., the average power harvested from the PLC additive noise is the highest in the morning, which makes the case \#1 in the morning as the best one in terms of offering the highest achievable data rates. Also, the WLC noise has an average power that does not change a lot during the day, thus the achievable data rate in case \#2 follows the same behavior.
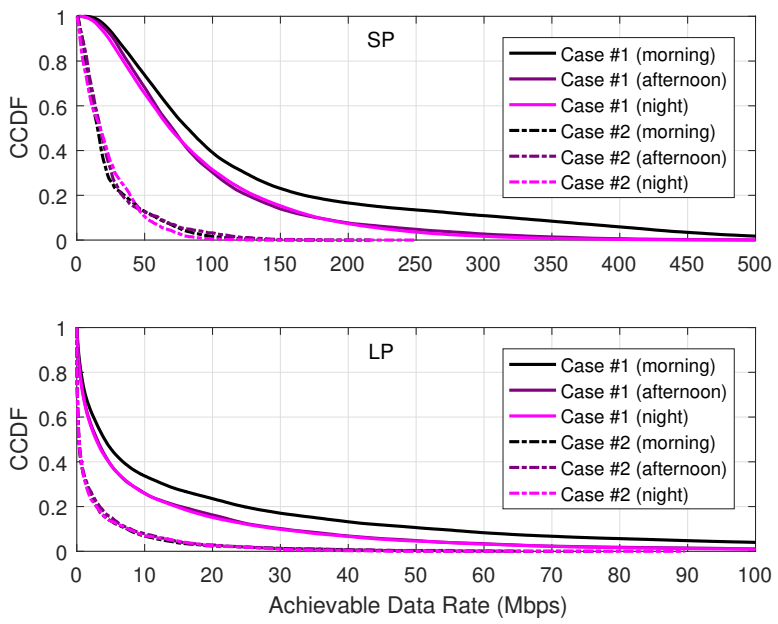

Fig. 7: Achievable data rates: The CCDF of per period of the day for case \#1 (continuous line) and case \#2 (dashed line).

\section{CONCLUSION}

This work has statistically evaluated the amount of energy that can be harvested from PLC and WLC additive noises. This evaluation was performed in terms of the average power. A practical perspective applied because data sets constituted by measures, which were obtained from urban residences and in different periods of the day (morning, afternoon and night), applied. The numerical results showed that the average power of the additive noise is higher in PLC than in the WLC medium. In fact, while $80 \%$ of the average power of the PLC additive noise is higher than $-9 \mathrm{dBmW}$, this value is around $-17 \mathrm{dBmW}$ in WLC one. The PLC additive noise yields more average power in the morning than in another period of the day, while such relation is not observed for the WLC additive noise.

Furthermore, the achievable data rates were analyzed using a set of channel estimates of the hybrid PLC-WLC channel and the transmission power harvested from the additive noise in both, PLC and WLC media. In this sense, two scenarios were taken into account by varying the distance between the WLC device and the power line outlet where the PLC device was connected, in a radius between 2 and $6 \mathrm{~m}$ as well as the two possible transmission directions (e.g., from PLC to WLC device and from PLC to WLC device) were evaluated. The numerical results have shown the potentialities of harvesting the existing wasted energy in the additive wireless and power line noises. Indeed, data rates from hundreds of kbps up to tens of Mbps can be achieved with the harvested energy from these additive noises. 


\section{REFERENCES}

[1] G. Y. Mao, G. Yu, and C. Zhong, "Energy consumption analysis of energy harvesting systems with power grid," IEEE Wireless Commun. Lett., vol. 2, no. 6, pp. 611-614, Dec. 2013, doi: 10.1109/WCL.2013.081913.130391.

[2] D. Mishra et al., "Smart RF energy harvesting communications: Challenges and opportunities," IEEE Commun. Mag., vol. 53, no. 4, pp. 70-78, Apr. 2015, doi: 10.1109/MCOM.2015.7081078.

[3] P. Kamalinejad et al., "Wireless energy harvesting for the Internet of Things," IEEE Commun. Mag., vol. 53, no. 6, pp. 102-108, Jun. 2015, doi: 10.1109/MCOM.2015.7120024.

[4] K. M. Rabie and B. Adebisi, "Enhanced amplify-and-forward relaying in non-Gaussian PLC networks," IEEE Access, vol. 5, pp. 4087-4094, Mar. 2017, doi: 10.1109/ACCESS.2017.2680599.

[5] K. Tashiro, H. Wakiwaka, S. Inoue, and Y. Uchiyama, "Energy harvesting of magnetic power-line noise," IEEE Trans. Magn., vol. 47, no. 10, pp. 4441-4444, Oct. 2011, doi: 10.1109/TMAG.2011.2158190.

[6] V. Fernandes, T. R. Oliveira, T. F. A. Nogueira, and M. V. Ribeiro, "Ambient energy harvesting: The potential of additive noise in urban and rural medium-voltage electric power networks," in Brazilian Telecommunications Symposium, Sep. 2019, doi: 10.14209/SBRT.2019.1570557222.

[7] V. Fernandes, H. V. Poor, and M. V. Ribeiro, "A hybrid power line/wireless dual-hop system with energy harvesting relay," IEEE Internet Things J., vol. 5, no. 5, pp. 4201-4211, Oct. 2018, doi: 10.1109/JIOT.2018.2860458.

[8] T. R. Oliveira, F. J. A. Andrade, A. A. M. Picorone, H. A. Latchman, S. L. Netto, and M. V. Ribeiro, "Characterization of hybrid communication channel in indoor scenario," J. Commun. and Inf. Syst., vol. 31, no. 1, pp. 224-235, 2016, doi: 10.14209/JCIS.2016.20.

[9] T. R. Oliveira, A. A. M. Picorone, C. B. Zeller, S. L. Netto, and M. V. Ribeiro, "On the statistical characterization of hybrid PLC-wireless channels," Electr. Pow. Syst. Res., no. 163, pp. 329-337, Aug. 2018, doi: 10.1016/J.EPSR.2018.07.004.

[10] L. R. Amado, E. S. C. Losqui, F. P. V. de Campos, A. A. M. de Medeiros, and M. V. Ribeiro, "Spectrum sensing for powering power line communications," in Brazilian Telecommunications Symposium, Sep. 2012, doi: 10.14209/SBRT.2012.218.

[11] J. M. Cioffi, Chapter 4: Multi-channel modulation, acessed in May 2018. [Online]. Available: http://web.stanford.edu/group/cioffi/book/chap4.pdf

[12] Measured Brazilian places, acessed in April 2018. [Online]. Available: https://sites.google.com/a/ifsudestemg.edu.br/thiagooliveira/plc

[13] T. R. Oliveira, C. A. G. Marques, W. A. Finamore, S. L. Netto, and M. V. Ribeiro, "A methodology for estimating frequency responses of electric power grids," Journal of Control, Automation and Electrical Systems, vol. 25, no. 6, pp. 720-731, 2014, doi: 10.1007/S40313-014-0151-5.

[14] L. G. da S. Costa, A. C. M. de Queiroz, B. Adebisi, V. L. R. da Costa, and M. V. Ribeiro, "Coupling for power line communications: A survey," J. Commun. and Inf. Syst., vol. 32, no. 1, 2017, doi: 10.14209/JCIS.2017.2.

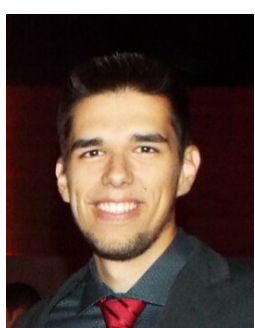

Victor Fernandes received the B.S. and M.Sc. degrees in Electrical Engineering from the Federal University of Juiz de Fora (UFJF), Brazil, in 2015 and 2017, respectively. He is a Professor at the Department of Electrical Engineering of the Centro de Ensino Superior de Juiz de Fora, Brazil, since 2019. $\mathrm{He}$ is currently working towards the D.Sc. degree in Electrical Engineering at UFJF since 2017. His research interests include signal processing, digital communication, power line communication, energy harvesting, smart grids, and the Internet of Things.

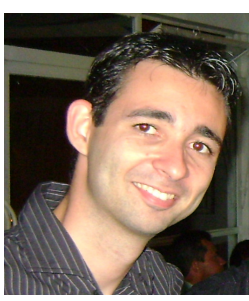

Thiago Rodrigues Oliveira received the B.S. (2007), M.Sc. (2010) and D.Sc. (2015) degrees from Federal University of Juiz de Fora (UFJF), all in Electrical Engineering. He has been a Professor at the Department of Electronics of the Federal Institute of Education, Science and Technology of the Southeast of Minas Gerais, Juiz de Fora, Brazil. His currently research works involve the following topics: digital signal processing, digital communication, internet of things, power line medium characterization.

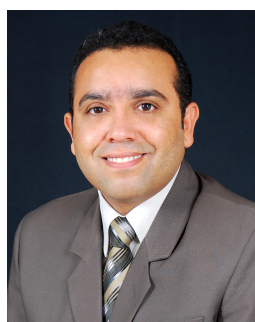

Moisés V. Ribeiro received the B.S. degree in Electrical Engineering from the Federal University of Juiz de Fora (UFJF), MG, Brazil, and M.Sc. and D.Sc. degrees in Electrical Engineering from the University of Campinas, SP, Brazil, in 1999, 2001 and 2005, respectively. He was a Visiting Scholar at the University of California in Santa Barbara, CA, USA, in 2004, Visiting Professor (2005-2007) and Assistant Professor (2007-2015) at UFJF. Since 2015, he has been an Associate Professor at UFJF. He co-founded Smarti9 LTD. and Wari LTD. in 2012 and 2015, respectively. He had served as the Secretary of the IEEE ComSoc TC-PLC.

Dr. Ribeiro was the recipient of Fulbright Visiting Professorship at Stanford University, Stanford, CA, USA, in 2011, and at Princeton University, Princeton, NJ, USA, in 2012. He was the General Chair of the 2010 IEEE ISPLC, 2013 IWSGC, SBrT 2015, and a Guest Co-Editor for Special Issues in the EURASIP Journal on Advances in Signal Processing and EURASIP Journal of Electrical and Computer Engineering. He was awarded Student Awards from 2001 IEEE IECON and 2003 IEEE ISIE, Winner of 2014 I2P Global Competition, Honorable Mention in 2014 Global Venture Labs Investment Competition, 3rd Place Prêmio Mineiro de Inovação 2014, Engie Brazil Innovation Award 2016, Unicamp Inventor Award in 2017 and 2018.

Dr. Ribeiro research interests include signal processing, power line communication, wireless communication, computational intelligence, internet of everything. In these fields, he has advised 38 graduate students and authored over 191 peer-reviewed papers, 9 book chapters, and hold 13 issued/pending patents. 